\title{
A Novel Recombinant Fusion Protein with Soluble PD-1 and TIM-3 Domains Effectively Binds to Cancer Cells
}

JING CHEN, J. WU1 AND J. CAO*

Clinical Research Center, The Second Affiliated Hospital, Zhejiang University School of Medicine, Hangzhou 310009, Zhejiang Province, ${ }^{1}$ Xuancheng Vocational and Technical College, Xuancheng 242000, Anhui Province, China

Chen et al.: PD-1/TIM-3 fusion protein binds to cancer cells 
In this investigation, a DNA sequence was designed and synthesized by joining coding regions of soluble programmed cell death-1 and T-cell immunoglobulin and mucin-3 domains and cloned into the lentiviral expression vector pLVX-IRES-ZsGreen1. After infection of the recombinant lentivirus into HEK $293 \mathrm{~T}$ cells, the recombinant fusion protein, designated as SP1T3, was successfully expressed as detected by Western blotting. Binding assay was performed by incubating the cell lysate with cancer cells, which have high expressional levels of PD-L1/2 and galectin 9 and subsequent fluorescent staining showed strong membranous signals compared to negative controls using cell lysate without SP1T3, suggesting specific binding of SP1T3 to these ligand-expressing cancer cells. The recombinant fusion protein SP1T3 provided a useful tool for studying the effect of dual-blockade of immune checkpoint and its potential in targeted immunotherapy of cancer, which needs further investigation.

Key words: Immune checkpoint, PD-1, TIM-3, neoplasms, targeted immunotherapy

Immune checkpoints, including stimulatory checkpoints and inhibitory checkpoints, are important modulators for immune responses, which are crucial for effective immunosurviallance while maintaining proper selftolerance ${ }^{[1]}$. Both checkpoints may be altered in cancer patients, which encounter weakened stimulatory checkpoint signaling and/or enhanced inhibitory checkpoint signaling. Specifically, cancer cells may exhibit aberrantly upregulated inhibitory checkpoint ligands, such as cytotoxic $\mathrm{T}$ lymphocyte-associated molecule-4 (CTLA-4) ligands, B7-1 (CD80) and B7-2 (CD86), programmed cell death receptor-1 (PD-1) ligands, PD-L1 and PD-L2, T-cell immunoglobulin and mucin-3 (TIM-3) ligand, galectin 9, resulting in immunoevasion and chemoresistance acquisition in cancer cells ${ }^{[2-4]}$. Inhibitory immune checkpoints are emerging targets for cancer immunotherapy, which have shown clinical success by blockade of inhibitory checkpoint molecules CTLA-4, PD-1 or PD-L1 in melanoma, non-small cell lung cancer, renal cell carcinoma and hematologic malignancies ${ }^{[5,6]}$ and more drugs are in clinical trials targeting other checkpoint molecules including TIM-3, LAG- $3^{[7]}$. However, antiCTLA4, antiPD-1 or antiPD-L1 used alone can achieve impressive clinical responses only in some patients, other patients may be naturally resistant, or get acquired resistance to blockade of one checkpoint interaction due to upregulated cancer cell expression of other checkpoint ligands, in other words, simultaneous upregulation of these ligands in cancer cells may occur $^{[8-10]}$. Improved therapeutic intervention for these patients need multiple-blockade of checkpoint interactions $^{[11]}$.

Checkpoint blockade can be achieved by blocking either the receptor on $\mathrm{T}$ cell surface or the ligand on cancer cell surface, such as nivolumab blocks PD-1 on

*Address for correspondence

E-mail: caoj@zju.edu.cn

May-June 2020
T cell or atezolizumab blocks PD-L1 on cancer cell. Although monoclonal antibody is the most popular molecule for the blocking purpose, a ligand-trap strategy was postulated, the native or engineered ligandbinding domain of receptor that has highly specific binding capacity can efficiently bind to and inactivate/ disable the ligand, as illustrated by VEGF-Trap (Aflibercept $)^{[12]}$ and FGF Trap (FP-1039) $)^{[13]}$, may also serve as block agents.

As coexpression of PD-L1 and galectin 9 is common in several kinds of cancer cells, along with more severely impaired tumor infiltrating lymphocyte (TIL) population $^{[14,15]}$, immunosuppression pathways in PD-1 and TIM-3 expressing T cells will be effectively blocked if the two ligands can be inactivated/ disabled simultaneously and it has been reported that immunotherapy with dual-checkpoint-targeting, especially PD-1 and TIM-3, is anticipated to be more effective than that with single-checkpoint-targeting ${ }^{[16]}$. For this purpose, a construct which encodes a recombinant fusion protein $\mathrm{SP} 1 \mathrm{~T} 3$ was designed as follows in this work, which included, a N-terminal Gaussia luciferase signal peptide for optimal expression and secretion of the mature protein in mammalian cells ${ }^{[17]}$, a soluble PD-1 domain (GenBank accession number NM_005018.2, nucleotides 129 to 578), an immunoglobulin $G$ hinge region (GenBank accession number JQ666008.1, nucleotides 4 to 72) as a flexible linker to maintain the structure and minimize the interference of the two domains, a soluble TIM-3

This is an open access article distributed under the terms of the Creative Commons Attribution-NonCommercial-ShareAlike 3.0 License, which allows others to remix, tweak, and build upon the work non-commercially, as long as the author is credited and the new creations are licensed under the identical terms 
domain (GenBank accession number NM_032782.4, nucleotides 318 to 860 ), and a $6 x$ His tag for detection. XhoI restriction sites were designed at both ends of sP1T3 for subsequent cloning (fig. 1). The above sP1T3 coding sequence was synthesized by Nanjing Genscript Biotechnology (Nanjing, Jiangsu, China), which was cloned into pUC57 plasmid (pUC57/sP1T3). The conceive of native human sequences of ligand-binding domains of soluble PD-1 (sPD-1) ${ }^{[18]}$ and soluble TIM3 (sTIM-3) $)^{[4]}$ with immunoglobulin hinge region as linker is to minimize possible immunogenic response in human. Moreover, it has been indicated in several works that recombinant soluble PD-1 could be an ideal tool to maintain or even enhance the function of T cells in cancer treatment ${ }^{[19-21]}$.

The plasmid pUC57/sP1T3 was digested by restriction endonuclease XhoI-HF (New England Biolabs Inc, Ipswich, MA, USA), and sP1T3 fragment (1146 bp) was separated by agarose gel (Sigma-Aldrich Corp, St. Louis, MO, USA) electrophoresis and excised from the gel, recovered by QIAquick Gel Extraction Kit (Qiagen $\mathrm{GmbH}$, Hilden, Germany), and ligated using T4 DNA ligase (Promega Corporation, Madison, WI, USA) overnight at $16^{\circ}$ with pLVX-IRES-ZsGreen1 vector (8204 bp, Clontech Laboratories, Inc., Mountain

A

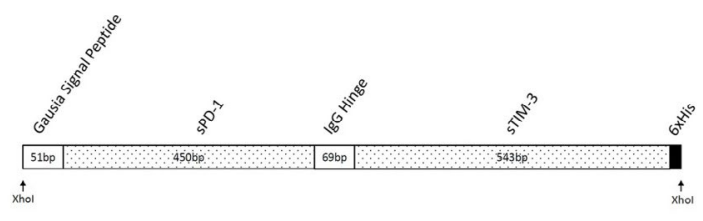

B

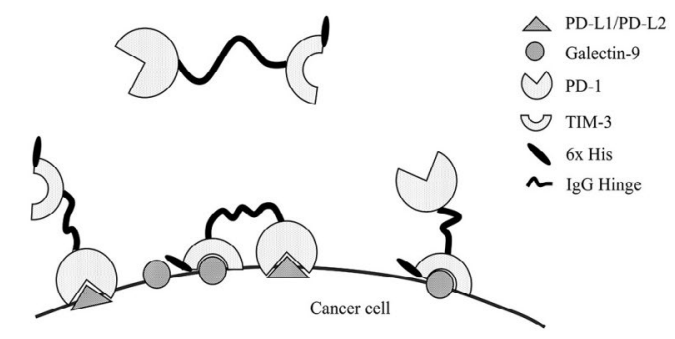

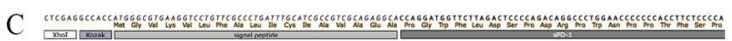

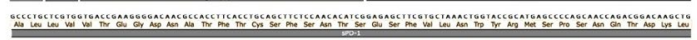

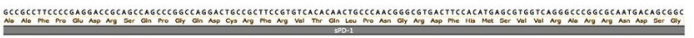

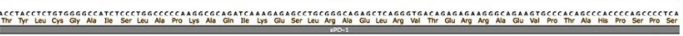

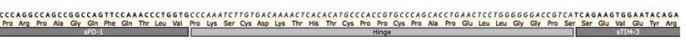

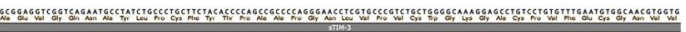

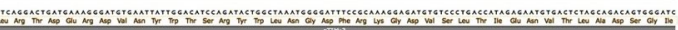

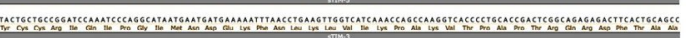

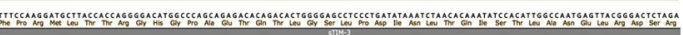

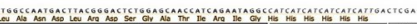

Fig. 1: Design of recombinant protein sP1T3

(A) Structure of sP1T3; (B) Illustration for dual binding capacity of sP1T3; (C) Detailed sequence information of sP1T3. A Kozak sequence precedes the coding sequence
View, CA, USA), which was digested by XhoI-HF and dephosphorylated by calf intestinal alkaline phosphatase (CIP, New England Biolabs Inc, Ipswich, MA, USA) to avoid self-ligation. The ligation mixture was transformed into DH5 $\alpha$ competent cells and spread on LB plate with ampicillin $(100 \mu \mathrm{g} / \mathrm{ml})$ and incubated overnight at $37^{\circ}$. Plasmids from transformants were extracted by Wizard plus SV minipreps DNA purification system (Promega Corporation, Madison, WI, USA), and digested by XhoI (expected to be 1146 bp and 8204 bp) and KpnI (expected to be 1419 bp, $1566 \mathrm{bp}$ and $6365 \mathrm{bp}$ ), respectively to identify expected construct, designated as pLVX-sP1T3-IRESZsGreen1 (fig. 2A).

HEK293T cells, purchased from the American Type Culture Collection (ATCC), were seeded into $100 \mathrm{~mm}$ cell culture dishes to approximately $80 \%$ confluency and co-transfected with plasmids pLVX-sP1T3-IRESZsGreen1, pMD2.G (envelope-VSV-G protein) and psPAX2 (packaging-Gag Pol Rev proteins) using Attractene Transfection Reagent Kit (Qiagen $\mathrm{GmbH}$, Hilden, Germany) according to the manufacturer's instruction. Supernatant containing viruses was collected after $48 \mathrm{~h}$ and $72 \mathrm{~h}$, and subsequently used to infect additional dishes of HEK293T cells for protein expression. At $48 \mathrm{~h}$ post-infection the cells were harvested and re-seeded into 96-well plates with $100 \mu \mathrm{l}$ per well at a density of 8 cells $/ \mathrm{ml}$. Wells with single cell were labeled by under a microscope. Cells with GFP expression identified by fluorescence microscope were designated as HEK293T-sP1T3 and propagated (fig. 2B).

Whole cell lysate of HEK293T-sP1T3 was prepared by ice-cold RIPA buffer $(150 \mathrm{mM} \mathrm{NaCl}, 100 \mathrm{mM}$ Tris $\mathrm{pH} 8.0,0.1 \%$ SDS, $1 \%$ Triton X-100, $1 \%$ sodium deoxycholate, $5 \mathrm{mM}$ EDTA and $10 \mathrm{mM} \mathrm{NaF}$ ) containing Complete ${ }^{\mathrm{TM}}$ protease inhibitor cocktail tablet (Santa Cruz Biotechnology, Dallas, Texas, USA). Protein concentration was quantified using Pierce BCA protein assay kit (ThermoFisher Scientific, Rockford, Illinois, USA) with absorbance at $562 \mathrm{~nm}$ measured by a microplate reader (SpectraMax M5, Molecular Devices, Sunnyvale, CA, USA). Protein samples of sP1T3 both from cell lysate and culture medium were prepared by mixing with $5 \mathrm{x}$ sample buffer (250 mM Tris- $\mathrm{HCl}$ pH 6.8, $10 \%$ SDS, $50 \%$ glycerol, $5 \%$ 2-hydroxy-1-ethanethiol, $0.5 \%$ bromophenol blue) at a ratio of $4: 1$, denatured for $5 \mathrm{~min}$ at $98^{\circ}$ followed by $5 \mathrm{~min}$ on ice, and subjected electrophoresis with $10 \%$ SDS-polyacrylamide gel containing 
$0.5 \%$ 2,2,2-trichloroethanol (Sigma-Aldrich Corp, St. Louis, MO, USA) for stain-free observation of protein bands under ultraviolet light, followed by transfer onto $0.45 \mathrm{~nm}$ PVDF membrane (Millipore Corporation, Bedford, MA, USA). The membrane was blocked with $5 \%$ skim milk (ThermoFisher Scientific, Rockford, Illinois, USA $)$ in TBS-T $(0.5 \mathrm{M} \mathrm{NaCl}, 0.1 \mathrm{M}$ Tris$\mathrm{HCl}, 0.1 \%$ Tween-20) for $1 \mathrm{~h}$ at room temperature and probed with HRP-conjugated 6x His Tag antibody (Hangzhou Hua An Biotechnology, Hangzhou, Zhejiang, China) with the dilution of 1:2000 in 5\% skim milk and incubated overnight at $4^{\circ}$ on a shaker. After three washes with TBS-T, Luminata ${ }^{\mathrm{TM}}$ Western HRP substrates (Millipore Corporation, Billerica, MA, USA) was applied and the signals were visualized and recorded by ChemiDoc ${ }^{\mathrm{TM}}$ MP Imaging System (v5.2.1, Bio-Rad Laboratories, Inc, Hercules, CA, USA). Shown as the result, sP1T3 was successfully expressed by HEK293T-sP1T3 cells and detectable in both whole cell lysate and culture medium (fig. 2C). The secreted form of recombinant $\mathrm{sP} 1 \mathrm{~T} 3$ in culture medium showed 2 distinct specific bands with apparent molecular sizes of approximately $90 \mathrm{kDa}$ and over $180 \mathrm{kDa}$, while intracellular sP1T3 showed one expected band at $42 \mathrm{kDa}$. Post-translational modifications such as glycosylation, which is common for secretory or membranous proteins for better functions, may contribute to such molecular weight differences between secreted form of sP1T3 and expected one $\mathrm{e}^{[22-24]}$.

Three cancer cell lines were screened for expression level of ligands for PD-1 and TIM-3 based on literature, colorectal cancer SW620 cells, breast cancer MDAMB-231 cells and prostate cancer PC-3 cells ${ }^{[25]}$. Total RNA of SW620, MDA-MB-231 and PC-3 cells (purchased from ATCC), were extracted using TRIZOL reagent (Life Technologies Corporation, Carlsbad, CA, USA) according to the manufacturer's instructions. And cDNA was prepared using $2 \mu \mathrm{g}$ total RNA, $0.4 \mathrm{mM}$ oligo (dT) ${ }_{18}, 0.8 \mathrm{mM}$ dNTPs, 200 units M-MLV Reverse Transcriptase (Promega Corporation, Madison, WI, USA) and 25 units Recombinant RNasin ${ }^{\circledR}$ Ribonuclease (Promega Corporation, Madison, WI, USA) to $25 \mu$ l final volume following the recommended procedure. PCR was performed with $1 \mu 1$ cDNA, $0.2 \mu 1$ GoTaq DNA Polymerase (Promega Corporation, Madison, WI, USA), $0.5 \mathrm{mM}$ dNTPs and $0.5 \mathrm{mM}$ specific forward and reverse primers (Table 1$)^{[26]}$ in total $20 \mu 1$ reaction system by following cycling conditions: denaturation at $95^{\circ}$ for $5 \mathrm{~min}$ followed by 30 cycles of $95^{\circ}$ for $30 \mathrm{~s}$ and then annealing at $54^{\circ}$ (for galectin-9) or $58^{\circ}$ (for PD-L1, PD-L2 and GAPDH) for $30 \mathrm{~s}$, elongation at $72^{\circ}$ for $30 \mathrm{~s}$, and an additional step of $72^{\circ}$ for $10 \mathrm{~min}$. The PCR product was

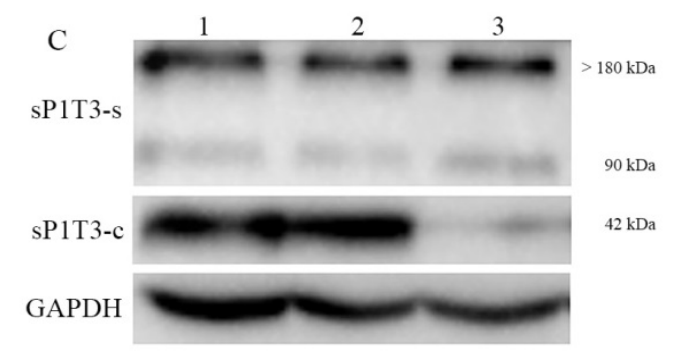

B
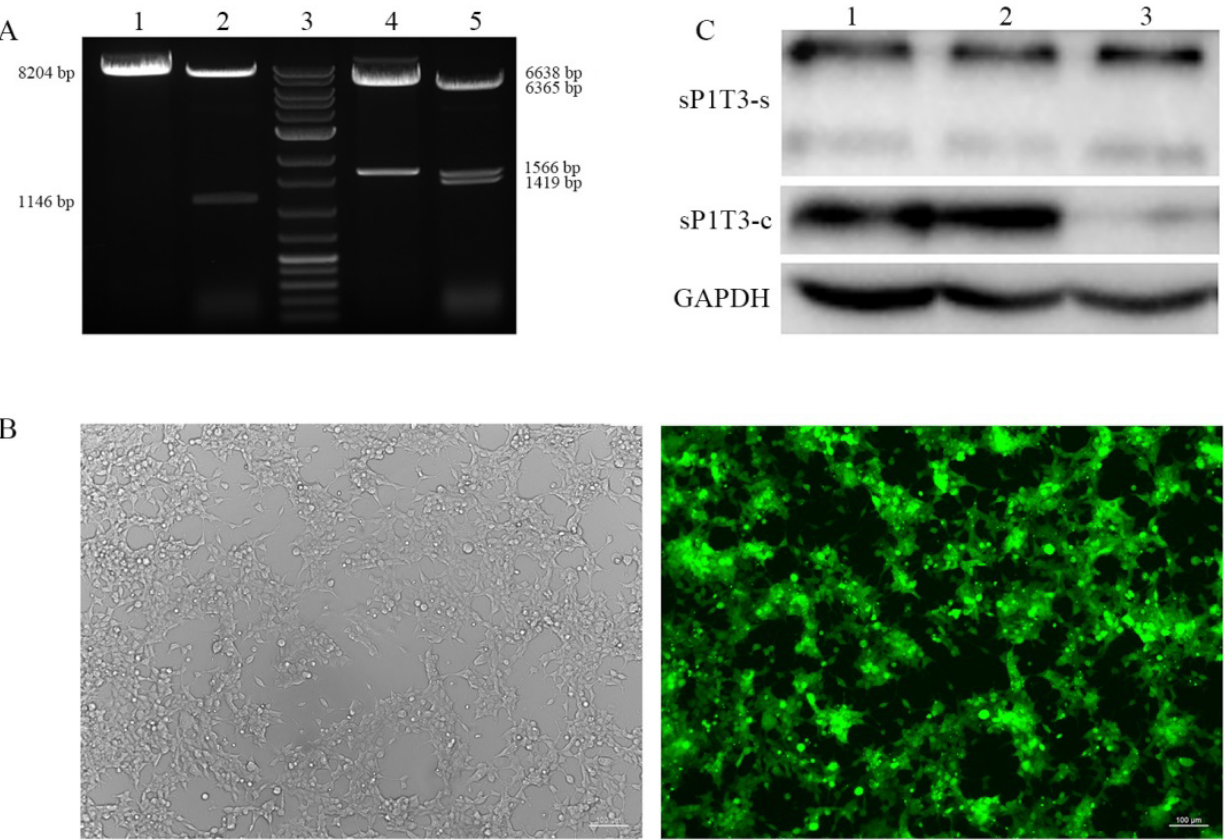

Fig. 2: Construction and expression of sP1T3 in HEK293T cells (A) Characterization of recombinant plasmid pLVX-IRES-ZsGreen1-sP1T3. Lane 1: pLVX-IRES-ZsGreen1, XhoI digested; Lane 2: pLVX-IRES-ZsGreen1-sP1T3, XhoI digested; Lane 3: 1 kb ladder plus marker; Lane 4: pLVX-IRES-ZsGreen1, KpnI digested; Lane 5: pLVX-IRES-ZsGreen1-sP1T3, KpnI digested. (B) HEK293T-sP1T3 cells under a microscope. Bright- field and fluorescent observation. Bar $=100 \mu \mathrm{m}$. (C) Expression of sP1T3 by three individual transfectant cell clones. sP1T3-s: secreted form of sP1T3 in concentrated supernatant of cell culture medium; sP1T3-c: sP1T3 in whole cell lysate 
analyzed by $1 \%$ agarose gel electrophoresis. SW620 cells showed very high level of galectin 9 (483 bp and $560 \mathrm{bp}$ ) expression but very low level of PD-L1 (133 bp) or PD-L2 (102 bp) expression. MDA-MB-231 cells showed lower level of galectin-9 expression but higher levels of PD-L1 and PD-L2 expression than SW620 cells did. Expression levels of these ligands in PC-3 cells were moderate when compared to those in the other two cells (fig. 3A).

To get higher concentration of sP1T3, cell lysate of HEK293T-sP1T3 was prepared for subsequent binding assay. One plate of HEK293T-sP1T3 cells cultured in $150 \mathrm{~mm}$ dishes with 90-100\% confluency were harvested and washed with ice-cold PBS (137 mM $\mathrm{NaCl}, 2.7 \mathrm{mM} \mathrm{KCl}, 10 \mathrm{mM} \mathrm{Na} \mathrm{HPO}_{4}, 2 \mathrm{mM} \mathrm{KH}_{2} \mathrm{PO}_{4}$ ), resuspended in $1 \mathrm{ml}$ PBS. Cells were then lysed by ultrasonication at $30 \%$ power and for $9.9 \mathrm{~s}$, repeated for three times, centrifuged at $13000 \mathrm{rpm}$ for $10 \mathrm{~min}$ at $4^{\circ}$, and the supernatant containing $\mathrm{SP} 1 \mathrm{~T} 3$ protein was collected. HEK293T cells were manipulated in the same manner for sP1T3-free lysate as negative control.

Cancer cells were seeded into a 24 -well plate and cultured for $24 \mathrm{~h}$, then the cells were washed three times with PBS for $5 \mathrm{~min}$ each time, and fixed by $4 \%$ formaldehyde $(300 \mu \mathrm{l} / \mathrm{well})$ for $20 \mathrm{~min}$ at room temperature. After fixation, the cells were washed for three times, followed by blocking with $300 \mu 15 \%$ BSA for $2 \mathrm{~h}$ at room temperature. Cell lysate with or without $\mathrm{sP} 1 \mathrm{~T} 3$ protein prepared above (with $3 \mathrm{mg} / \mathrm{ml}$ total protein content each), with PBS as blank control, was then added onto the cells and incubated overnight at $4^{\circ}$ for binding. After wash, cells were incubated with anti-6x His tag ${ }^{\circledR}$ antibody (AD1.1.10, DyLight ${ }^{\circledR} 650$, Ex: $654 \mathrm{~nm}$, Em: $673 \mathrm{~nm}$, Abcam, Cambridge, MA, USA) 1:2000 diluted in BSA for $2 \mathrm{~h}$ protected from light at room temperature. Hoechst 33342 (SigmaAldrich Corp, St. Louis, MO, USA) was used to stain the nuclei. The signals were observed under an inverted

\section{TABLE 1: PRIMERS SEQUENCE}

\begin{tabular}{ll}
\hline Primer Name & Sequence \\
\hline PD-L1-Forward & 5'-ATGCCTTGGTGTAGCACTGAC-3' \\
PD-L1-Reverse & 5'-GCTGGATTACGTCTCCTCCAAATG-3' \\
PD-L2-Forward & 5'-CTGTGTGTTCTGGAATACTCACGTG-3' \\
PD-L2-Reverse & 5'-ATGTGAAGCAGCCAAGTTGGATG-3' \\
galectin-9-Forward & 5'-CGTCAATGGCTCTGTGCAGCTGTC-3' \\
galectin-9-Reverse & 5'-AGATCCACACTGAGAAGCTCTGGC-3' \\
GAPDH-Forward & 5'-CTTAGCACCCCTGGCCAAG-3' \\
GAPDH-Reverse & 5'-GATGTTCTGGAGAGCCCCG-3' \\
\hline
\end{tabular}

Sequence of specific forward and reverse primers used in RT-PCR with respective annealing temperature as follows, PD-L1: $58^{\circ}$, PDL2: $58^{\circ}$, galectin-9: $54^{\circ}$, GAPDH: $54^{\circ}$. GAPDH: Glyceraldehyde-3phosphate dehydrogenase 
checkpoint-targeting protein and further validation for its potential use in immunotherapy of cancer.

\section{Acknowledgements:}

The authors thank Muhammad Umar Ali for his help in virus packaging and infection.

\section{REFERENCES}

1. Sharpe AH. Introduction to checkpoint inhibitors and cancer immunotherapy. Immunol Rev 2017;276(1):5-8.

2. Black M, Barsoum IB, Truesdell P, Cotechini T, MacdonaldGoodfellow SK, Petroff M, et al. Activation of the PD-1/PDL1 immune checkpoint confers tumor cell chemoresistance associated with increased metastasis. Oncotarget 2016;7(9):10557-67.

3. Anderson AC. Tim-3: an emerging target in the cancer immunotherapy landscape. Cancer Immunol Res 2014;2(5):393-8.

4. Sabatos CA, Chakravarti S, Cha E, Schubart A, Sanchez-Fueyo A, Zheng XX, et al. Interaction of Tim-3 and Tim-3 ligand regulates $T$ helper type 1 responses and induction of peripheral tolerance. Nat Immunol 2003;4(11):1102-10.

5. Sledzinska A, Menger L, Bergerhoff K, Peggs KS, Quezada SA. Negative immune checkpoints on $\mathrm{T}$ lymphocytes and their relevance to cancer immunotherapy. Mol Oncol 2015;9(10):1936-65.

6. Pardoll DM. The blockade of immune checkpoints in cancer immunotherapy. Nat Rev Cancer 2012;12(4):252-64.

7. Marin-Acevedo JA, Dholaria B, Soyano AE, Knutson KL, Chumsri S, Lou Y. Next generation of immune checkpoint therapy in cancer: new developments and challenges. J Hematol Oncol 2018;11(1):39.

8. Koyama S, Akbay EA, Li YY, Herter-Sprie GS, Buczkowski $\mathrm{KA}$, Richards WG, et al. Adaptive resistance to therapeutic PD-1 blockade is associated with upregulation of alternative immune checkpoints. Nat Commun 2016;7:10501.

9. Romero D. Immunotherapy: PD-1 says goodbye, TIM-3 says hello. Nat Rev Clin Oncol 2016;13(4):202-3.

10. Marcucci F, Rumio C, Corti A. Tumor cell-associated immune checkpoint molecules - Drivers of malignancy and stemness. Biochim Biophys Acta 2017;1868(2):571-83.

11. Nirschl CJ, Drake CG. Molecular pathways: coexpression of immune checkpoint molecules: signaling pathways and implications for cancer immunotherapy. Clin Cancer Res 2013;19(18):4917-24.

12. Teng LS, Jin KT, He KF, Zhang J, Wang HH, Cao J. Clinical applications of VEGF-trap (aflibercept) in cancer treatment. J Chin Med Assoc 2010;73(9):449-56.
13. Tolcher AW, Papadopoulos KP, Patnaik A, Wilson K, Thayer $\mathrm{S}$, Zanghi J, et al. A phase I, first in human study of FP-1039 (GSK3052230), a novel FGF ligand trap, in patients with advanced solid tumors. Ann Oncol 2016;27(3):526-32.

14. Li $\mathrm{X}, \mathrm{Hu} \mathrm{W}$, Zheng $\mathrm{X}$, Zhang $\mathrm{C}$, Du $\mathrm{P}$, Zheng $\mathrm{Z}$, et al. Emerging immune checkpoints for cancer therapy. Acta Oncol 2015;54(10):1706-13.

15. Kim JE, Patel MA, Mangraviti A, Kim ES, Theodros D, Velarde E, et al. Combination Therapy with Anti-PD-1, AntiTIM-3, and Focal Radiation Results in Regression of Murine Gliomas. Clin Cancer Res 2017;23(1):124-36.

16. Hellmann MD, Friedman CF, Wolchok JD. Combinatorial Cancer Immunotherapies. Adv Immunol 2016;130:251-77.

17. Knappskog S, Ravneberg H, Gjerdrum C, Trosse C, Stern B, Pryme IF. The level of synthesis and secretion of Gaussia princeps luciferase in transfected $\mathrm{CHO}$ cells is heavily dependent on the choice of signal peptide. J Biotechnol 2007;128(4):705-15.

18. Nielsen C, Ohm-Laursen L, Barington T, Husby S, Lillevang ST. Alternative splice variants of the human PD-1 gene. Cell Immunol 2005;235(2):109-16.

19. Pan Z, Di S, Shi B, Jiang H, Shi Z, Liu Y, et al. Increased antitumor activities of glypican-3-specific chimeric antigen receptor-modified $\mathrm{T}$ cells by coexpression of a soluble PD1-CH3 fusion protein. Cancer Immunol Immunother 2018;67(10):1621-34.

20. Gu D, Ao X, Yang Y, Chen Z, Xu X. Soluble immune checkpoints in cancer: production, function and biological significance. J Immunother Cancer 2018;6(1):132.

21. Bartee MY, Dunlap KM, Bartee E. Tumor-Localized Secretion of Soluble PD1 Enhances Oncolytic Virotherapy. Cancer Res 2017;77(11):2952-63.

22. Stanley P. Golgi glycosylation. Cold Spring Harb Perspect Biol 2011;3(4):23.

23. Tokhtaeva E, Mareninova OA, Gukovskaya AS, Vagin $\mathrm{O}$. Analysis of $\mathrm{N}$ - and O-Glycosylation of Lysosomal Glycoproteins. Methods Mol Biol 2017;1594:35-42.

24. Butler M, Spearman M. The choice of mammalian cell host and possibilities for glycosylation engineering. Curr Opin Biotechnol 2014;30:107-12.

25. Grenga I, Donahue RN, Lepone LM. PD-L1 and MHC-I expression in 19 human tumor cell lines and modulation by interferon-gamma treatment. $\mathrm{J}$ Immunother Cancer 2014;2(Suppl 3):102.

26. Kageshita T, Kashio Y, Yamauchi A, Seki M, Abedin MJ, Nishi $\mathrm{N}$, et al. Possible role of galectin-9 in cell aggregation and apoptosis of human melanoma cell lines and its clinical significance. Int J Cancer 2002;99(6):809-16.

27. Postow MA. Managing immune checkpoint-blocking antibody side effects. Am Soc Clin Oncol Educ 2015;4:76-83. 